\title{
MPC for tracking periodic references
}

\author{
D. Limon, M. Pereira, D. Muñoz de la Peña, T. Alamo, C. N. Jones, M. N. Zeilinger
}

\begin{abstract}
In this paper a new model predictive controller for tracking arbitrary periodic references is presented. The proposed controller is based on a single layer that unites dynamic trajectory planning and control. A design procedure to guarantee that the closed loop system converges asymptotically to the optimal admissible periodic trajectory while guaranteeing constraint satisfaction is provided. In addition, the constraints of the optimization problem solved by the controller do not depend on the reference, allowing for sudden changes in the reference without loosing feasibility. The properties of the proposed controller are demonstrated with a simulation example of a ball and plate system.
\end{abstract}

Keywords-Predictive control, tracking, periodic references, stability.

\section{INTRODUCTION}

Model predictive control (MPC) is one of the most important control techniques used in industry to operate multivariable constrained systems. The problem of designing stabilizing model predictive control (MPC) schemes to regulate a system to a certain equilibrium point, typically the origin, has been widely studied, see e.g. [1], [2].

In this paper, we focus on the problem of tracking periodic references, which appear naturally in important control problems such as repetitive control [3], periodic systems [4], [5] or economic operation of complex systems [6]. In [7] a class of output feedback MPC for nonlinear discrete-time systems is proposed to solve the problem of tracking exogenous signals (and asymptotically rejecting disturbances) generated by systems with known dynamics. In [8] a predictive controller for the offset-free tracking of reference signals generated by arbitrary dynamics is proposed. This controller ensures that the tracking error tends to zero, but recursive feasibility and stability of the closed-loop system is not ensured in case of changing references. In [9] it is shown that the reference look-ahead action of the MPC plays an important role in the periodic reference tracking problem.

One relevant issue in tracking is that the reference trajectory may not be reachable by the constrained system. In order to deal with this problem, a hierarchical architecture is often adopted: a trajectory planner decides the optimal reachable trajectory, which is provided to an MPC controller as a trajectory target. In order to guarantee closedloop stability, a terminal constraint that depends on this reference signal is added. This constraint may lead to a loss of feasibility if a sudden change in the reference takes place [10].

In this paper we propose a different strategy based on a single layer that unites dynamic trajectory planning and control and is able to take into account arbitrary references. The proposed scheme extends the method presented in [11], [12] for tracking constant set-points to periodic references and is based on augmenting the decision variables

D. Limon, M. Pereira, D. Muñoz de la Peña and T. Alamo are with the Departamento de Ingeniería de Sistemas y Automática, Escuela Superior de Ingenieros, Universidad de Sevilla, Sevilla, Spain, e-mail: $\{$ dlm,mpereiram,dmunoz,talamo\}@us.es

C. N. Jones is in the Automatic Control Laboratory, EPFL, Lausanne, Switzerland, e-mail: \{colin.jones\}@epfl.ch

M. N. Zeilinger is with the Department of Electrical Engineering and Computer Sciences, University of California, Berkeley, CA 94720, USA, email: mzeilinger@berkeley.edu

The research leading to these results has received funding from the MINECO-Spain and FEDER under project DPI2013-48243-C2-2-R, the University of Seville under contracts 2014/425 and 2014/758 and the European Research Council under the European Unions Seventh Framework Programme (FP/2007- 2013)/ ERC Grant Agreement n. 307608. with a set of auxiliary variables that describe a future, periodic and admissible trajectory. The cost function penalizes both the tracking error of the predicted trajectory to the planned reachable one, and the deviation of the planned reachable trajectory to the target periodic reference. A design procedure to guarantee that the closed loop system converges asymptotically to the optimal reachable periodic trajectory while guaranteeing constraint satisfaction and recursive feasibility is provided. In addition, the constraints of the optimization problem solved by the controller do not depend on the reference, allowing for sudden changes in the reference without loosing feasibility. The properties of the proposed controller are demonstrated with a simulation example of a ball and plate system.

\section{Notation}

Bold letters are used to denote a sequence of $T$ values of a trajectory, i.e. $\mathbf{z}=\{z(0), \cdots, z(T-1)\} . \mathbf{z}(\theta)$ denotes the sequence $\mathbf{z}(\theta)=\{z(0 ; \theta), \cdots, z(T-1 ; \theta)\}$. If the cardinality of a sequence is not $T$, then the sequence is denoted as $\mathbf{z}_{N}(\theta)$ where $N$ is the cardinal. $\mathbb{I}_{[a, b]}$ denotes the set of integer numbers contained in the interval $[a, b]$, that is $\mathbb{I}_{[a, b]}=\{a, a+1, \cdots, b\}$.

\section{PROBLEM FORMULATION}

Consider a discrete time linear system described by the following state-space model

$$
\begin{array}{ll}
x(k+1) & =A x(k)+B u(k) \\
y(k) & =C x(k)+D u(k)
\end{array}
$$

where $x(k) \in R^{n}, u(k) \in R^{m}$ and $y(k) \in R^{p}$ are the state, input and output of the system at time step $k$.

Assumption 1: It is assumed that the pair $(A, B)$ is controllable and $(C, A)$ is observable.

From this assumption it can be proved that there exists an integer $n_{c} \geq n$ such that the following matrices

$$
\begin{gathered}
{\left[A^{n_{c}-1} B, \ldots, A B, B\right]} \\
{\left[C^{T},(C A)^{T}, \ldots,\left(C A^{n_{c}-1}\right)^{T}\right]}
\end{gathered}
$$

are full row rank.

The controller must ensure that the closed-loop system satisfies the hard constraints $(x(k), u(k)) \in \mathcal{Z}$ where set $\mathcal{Z}$ is a convex and compact polyhedron that contains the origin.

The control objective is to steer the output $\mathbf{y}$ as close as possible to an exogenous periodic reference $\mathbf{r}$ with period $T$. Since no assumptions on the provided reference signal are made, there may not exist a control law capable of steering the system to this reference signal. This can be a consequence of the limits imposed by the constraints and/or by the dynamics. In this case the reference is said to be unreachable.

If the reference is unreachable, then the controller cannot steer the output signal to the given reference. In this case the control goal is to steer the output to a reachable periodic trajectory that optimizes a certain criterion, which is referred to as the optimal reachable trajectory. In this paper, the optimal reachable trajectory is chosen such that the sum of the weighted squared error in a period $T$ is 
minimized. This trajectory is defined by the following optimization problem:

$$
\begin{aligned}
\min _{y^{r}, x^{r}, \mathbf{u}^{r}} & V_{p}\left(\mathbf{r} ; x^{r}, \mathbf{u}^{r}\right) \\
\text { s.t. } & x^{r}(j+1)=A x^{r}(j)+B u^{r}(j) \\
& y^{r}(j)=C x^{r}(j)+D u^{r}(j) \\
& \left(x^{r}(j), u^{r}(j) \in \mathcal{Z}^{c}\right. \\
& x^{r}(T)=x^{r}(0)
\end{aligned}
$$

where the set $\mathcal{Z}^{c}$ is a closed polyhedron contained into the relative interior of $\mathcal{Z}^{1}$ and

$$
V_{p}\left(\mathbf{r} ; x^{r}, \mathbf{u}^{r}\right)=\sum_{j=0}^{T-1}\left\|y^{r}(j)-r(j)\right\|_{S}^{2}
$$

The solution to this optimization problem defines the initial state and sequence of inputs of the optimal reachable trajectory $\left(x^{\circ}, \mathbf{u}^{\circ}\right)$. If the signal $\mathbf{r}$ is known and periodic with period $T$, then the solution of the optimization problem (2) does not depend on the time instant in which the periodic reference is evaluated. The optimal reachable trajectory $\left(\mathbf{y}^{\circ}, \mathbf{x}^{\circ}, \mathbf{u}^{\circ}\right)$ is obtained from the periodic extension of the solution of (2).

Note that if the reference $\mathbf{r}$ is unreachable, there exists an error between the optimal reachable trajectory and the reference to be tracked. We denote this cumulative error as

$$
V_{p}^{\circ}(\mathbf{r})=V_{p}\left(\mathbf{r} ; x^{\circ}, \mathbf{u}^{\circ}\right)
$$

Assumption 2: The optimization problem (2) is strictly convex.

Assumption 2 implies that the solution of the optimization problem is unique. Strict convexity can be checked easily since this is a quadratic programming problem.

The control objective is to design a state feedback tracking control law $u(k)=\kappa(x(k), \mathbf{r}(k))$ such that given a periodic reference $\mathbf{r}(k)$, the closed-loop system satisfies the constraints, is stable and converges to the optimal reachable trajectory. At each time step $k$, the periodic reference signal $\mathbf{r}(k)$ used to define the controller is different because the initial time of the sequence changes. With a slight abuse of notation, we define $\mathbf{r}$ as the target periodic reference, and $\mathbf{r}(k)$ the reference fed to the controller which takes into account the time shift.

Standard tracking schemes are usually based on a hierarchical architecture in which a trajectory planner computes the optimal reachable trajectory which is then used by a MPC as a target reference. This implies that the MPC controller depends on this optimal trajectory and that two different optimization problems have to be solved.

\section{PROPOSED CONTROLLER}

The proposed controller combines the trajectory planner and the MPC for tracking in a single optimization problem in which the decision variables are a planned reachable trajectory defined by its initial state $x^{r}$ and the corresponding sequence of inputs $\mathbf{u}^{r}$ as well as the sequence of future control inputs $\mathbf{u}_{N}$. The optimization problem minimizes the cost function $V_{N}\left(x, \mathbf{r} ; x^{r}, \mathbf{u}^{r}, \mathbf{u}_{N}\right)$, where the parameters $(x, \mathbf{r})$ stand for the current state and expected reference signal respectively.

$$
V_{N}\left(x, \mathbf{r} ; x^{r}, \mathbf{u}^{r}, \mathbf{u}_{N}\right)=V_{t}\left(x ; x^{r}, \mathbf{u}^{r}, \mathbf{u}_{N}\right)+V_{p}\left(\mathbf{r} ; x^{r}, \mathbf{u}^{r}\right)
$$

${ }^{1}$ The reason for considering a tighter constraint set $\mathcal{Z}^{c}$ is to avoid the possible loss of controllability when the constraints are active [13]. where

$$
\begin{aligned}
& V_{t}\left(x ; x^{r}, \mathbf{u}^{r}, \mathbf{u}_{N}\right)=\sum_{i=0}^{N-1}\left\|x(i)-x^{r}(i)\right\|_{Q}^{2}+\left\|u(i)-u^{r}(i)\right\|_{R}^{2} \\
& V_{p}\left(\mathbf{r} ; x^{r}, \mathbf{u}^{r}\right)=\sum_{i=0}^{T-1}\left\|y^{r}(i)-r(i)\right\|_{S}^{2}
\end{aligned}
$$

with $N \leq T$. The term $V_{t}\left(x ; x^{r}, \mathbf{u}^{r}, \mathbf{u}_{N}\right)$ penalizes the tracking error of the open-loop predicted trajectory with respect to the planned reachable reference along the prediction horizon $N$. The term $V_{p}\left(\mathbf{r} ; x^{r}, \mathbf{u}^{r}\right)$ penalizes the error between the planned reachable trajectory and the reference to be tracked predicted for one period $T$.

In order to evaluate the MPC for tracking periodic references, the following optimization problem $P_{N}(x, \mathbf{r})$ is solved at each sampling time:

$$
\begin{aligned}
\min _{x^{r}, \mathbf{u}^{r}, \mathbf{u}_{N}} & V_{N}\left(x, \mathbf{r} ; x^{r}, \mathbf{u}^{r}, \mathbf{u}_{N}\right) \\
\text { s.t. } & x(0)=x \\
& x(i+1)=A x(i)+B u(i) \quad i \in \mathbb{I}_{[0, N-1]} \\
& y(i)=C x(i)+D u(i) \quad i \in \mathbb{I}_{[0, N-1]} \\
& (x(i), u(i)) \in \mathcal{Z} \quad i \in \mathbb{I}_{[0, N-1]} \\
& x^{r}(0)=x^{r} \\
& x^{r}(i+1)=A x^{r}(i)+B u^{r}(i) \quad i \in \mathbb{I}_{[0, T-1]} \\
& y^{r}(i)=C x^{r}(i)+D u^{r}(i) \quad i \in \mathbb{I}_{[0, T-1]} \\
& \left(x^{r}(i), u^{r}(i)\right) \in \mathcal{Z}^{c} \quad i \in \mathbb{I}_{[0, T-1]} \\
& x^{r}(0)=A x^{r}(T-1)+B u^{r}(T-1) \\
& x(N)=x^{r}(N)
\end{aligned}
$$

The optimal solution of this optimization problem is denoted $\left(x^{r *}, \mathbf{u}^{r *}, \mathbf{u}_{N}^{*}\right)$. The variables $\mathbf{x}_{N}^{*}(x, \mathbf{r}), \mathbf{y}_{N}^{*}(x, \mathbf{r})$ denote the optimal predicted trajectories of the states and outputs of the system and $\mathbf{x}^{r *}(x, \mathbf{r}), \mathbf{y}^{r *}(x, \mathbf{r})$ denote the optimal planned reachable trajectories of the states and outputs of the system. The control law is given by the first input of the optimal reachable predicted trajectory, $\kappa_{N}(x(k), \mathbf{r}(k))=u_{N}^{*}(0 ; k)$.

Constraints (4b-4d) define the predicted trajectories of the system starting from the current state. Constraints $(4 \mathrm{f}-4 \mathrm{~h})$ define the planned reachable reference starting from the free initial state $x^{r}$. Constraints (4e) and (4i) include the state and input constraints for both the predicted states and the planned reachable reference. In addition, two terminal constraints are included to guarantee closed-loop convergence to the optimal reachable trajectory. Constraint $(4 \mathrm{j})$ is added to enforce that the reachable trajectory is periodic, while constraint (4k) guarantees that the terminal state of the predicted trajectory of the plant reaches the planned reachable trajectory at the end of the prediction horizon.

It is important to point out that the set of constraints of this optimization problem does not depend on the reference signal $\mathbf{r}$. The domain of attraction $\mathcal{X}_{N}$ is defined as the set of states that can admissibly reach any reachable periodic trajectory in $N$ steps, and in general is large if compared with the set of states that can admissibly reach a particular reachable periodic trajectory. The controller can be used to track any target reference, including high frequency signals.

\section{STABILITY ANALYSIS}

In this section we study the closed-loop properties of the proposed control law. In particular we prove that the output converges asymptotically to the optimal reachable trajectory and that the controller maintains feasibility even in the presence of sudden changes in the target reference. To this end, we make use of the following slightly modified Lyapunov theorem, see [1]: 
Theorem 1: Consider an autonomous system $z(k+1)=f(z(k))$ where $z(k) \in \mathbb{R}^{n}$. Let $\Gamma$ be a positive invariant set and $\Omega \subseteq \Gamma$ be a compact set, both including the origin as an interior point. If there exists a function $W: \mathbb{R}^{n} \rightarrow \mathbb{R}^{+}$and suitable $\mathcal{K}_{\infty}$-class functions $\alpha_{1}, \alpha_{2}, \alpha_{3}$ such that

$$
\begin{aligned}
& (i) W(z(k)) \geq \alpha_{1}(\|z(k)\|), \quad \forall z(k) \in \Gamma \\
& (i i) W(z(k)) \leq \alpha_{2}(\|z(k)\|), \quad \forall z(k) \in \Omega \\
& (i i i) W(z(k+1))-W(z(k)) \leq-\alpha_{3}(\|z(k)\|), \quad \forall z(k) \in \Gamma
\end{aligned}
$$

then $W(\cdot)$ is called a Lyapunov function in $\Gamma$ and the origin is asymptotically stable for all initial states in $\Gamma$.

In the following theorem, we will use this result to prove the existence of a Lyapunov function and then derive the asymptotic stability of the optimal trajectory.

Theorem 2: Assume that system (1) satisfies Assumptions 1 and 2, the weighting matrices $Q$ and $R$ are positive definite and the prediction horizon is such that $N \geq n_{c}$. Then system (1) controlled by the proposed control law is recursively feasible and the optimal reachable trajectory $\mathbf{x}^{\circ}$ given by $\left(x^{\circ}, \mathbf{u}^{\circ}\right)$ is asymptotically stable with region of attraction $\mathcal{X}_{N}$, i.e. the closed loop system is stable and $x(k)$ converges asymptotically to $\mathbf{x}^{\circ}(k)$ for all $x(0) \in \mathcal{X}_{N}$.

Proof: Asymptotic stability will be proved by demonstrating that for the system that models the error between the state of the reachable optimal trajectory and the closed loop trajectory of the system, $z(k)=$ $x(k)-x^{\circ}(k)$, the function

$$
W(z(k))=W\left(x(k)-x^{\circ}(k)\right)=V_{N}^{*}(x(k), \mathbf{r}(k))-V_{p}^{\circ}(\mathbf{r})
$$

satisfies the conditions of Theorem 1 in the region $\mathcal{X}_{N}$ and provides a Lyapunov function. This function is defined as the difference between the optimal cost of the MPC problem at time $k, V_{N}^{*}(x(k), \mathbf{r}(k))$, and the cost value of the optimal reachable trajectory defined in (3). To simplify the notation, we have dropped the dependence of function $W(\cdot)$ on the target reference $\mathbf{r}$. In addition, we will not use the error $z(k)$ in the following derivations, but its definition, $x(k)-x^{\circ}(k)$.

In what follows, $y(i ; k), x(i ; k)$ are the output and state predicted at time $i$ applying $\mathbf{u}(k)$ from the initial state $x(k) ; y^{r}(i ; k), x^{r}(i ; k)$ are the output and state of the planned reachable reference at time $i$ applying $\mathbf{u}^{r}(k)$ from the initial state $x^{r}(k) ; y^{\circ}(i ; k), x^{\circ}(i ; k)$ are the output and state of the optimal reachable reference $i$ applying $\mathbf{u}^{\circ}(k)$ from the initial state $x^{\circ}(k)$.

First, we will prove that the region $\mathcal{X}_{N}$ is a positive invariant set for the system in closed-loop with the proposed controller, and hence, also for $x(k)-x^{\circ}(k)$. Consider the shifted sequences

$$
\begin{aligned}
& \mathbf{u}_{N}^{s}(k)=\left\{u_{N}^{*}(1 ; k-1), \cdots, u_{N}^{*}(N-1 ; k-1), u_{N}^{r *}(N ; k-1)\right\} \\
& x^{r s}(k)=x^{r *}(1 ; k-1) \\
& \mathbf{u}^{r s}(k)=\left\{u^{r *}(1 ; k-1), \cdots, u^{r *}(T-1 ; k-1), u^{r *}(0 ; k-1)\right\}
\end{aligned}
$$

Taking into account that the optimal solution at time $k-1$ is feasible by definition, it is easy to prove that the shifted sequences are also feasible at time $k$. Note that the constraints of problem (4) do not depend on the reference, so this is true even in the presence of arbitrary changes of $\mathbf{r}$.

Next, we will prove that the proposed Lyapunov function satisfies the conditions of Theorem 1 .

Condition (i): From the definition of $W(\cdot)$ we have that

$$
\begin{aligned}
W\left(x(k)-x^{\circ}(k)\right) & =\sum_{i=0}^{N-1}\left\|\left(x^{*}(i ; k)-x^{r *}(i ; k)\right)\right\|_{Q}^{2} \\
& +\left\|\left(u^{*}(i ; k)-u^{r *}(i ; k)\right)\right\|_{R}^{2} \\
& +V_{p}\left(\mathbf{r}(k), x^{r *}(k), \mathbf{u}^{r *}(k)\right)-V_{p}^{\circ}(\mathbf{r}) \\
& \geq\left\|x(k)-x^{r *}(k)\right\|_{Q}^{2} \\
& +V_{p}\left(\mathbf{r}(k), x^{r *}(k), \mathbf{u}^{r *}(k)\right)-V_{p}^{\circ}(\mathbf{r})
\end{aligned}
$$

From the strictly convexity of optimization problem (2), there exists a $\pi_{1}>0$ such that

$$
V_{p}\left(\mathbf{r}(k), x^{r *}(k), \mathbf{u}^{r *}(k)\right)-V_{p}^{\circ}(\mathbf{r}) \geq \pi_{1}\left\|\left(x^{r *}(k)-x^{\circ}(k)\right)\right\|^{2}
$$

and therefore, since $Q$ is positive definite,

$$
\begin{aligned}
W\left(x(k)-x^{\circ}(k)\right) & \geq \lambda_{\min }(Q)\left\|\left(x(k)-x^{r *}(k)\right)\right\|^{2} \\
& +\pi_{1}\left\|\left(x^{r *}(k)-x^{\circ}(k)\right)\right\|^{2} \\
& \geq \alpha_{1}\left(\left\|\left(x(k)-x^{r *}(k)\right)\right\|^{2}\right. \\
& \left.+\left\|\left(x^{r *}(k)-x^{\circ}(k)\right)\right\|^{2}\right) \\
& \geq \frac{\alpha_{1}}{2}\left\|\left(x(k)-x^{\circ}(k)\right)\right\|^{2}
\end{aligned}
$$

with $\alpha_{1}=\min \left\{\lambda_{\min }(Q), \pi_{1}\right\}>0$.

Condition (ii): Since the optimal reachable trajectory is contained in the relative interior of the set of constraints $\mathcal{Z}$, there exists a sufficiently small neighborhood $\Upsilon$ such that for all $\left(x(k)-x^{\circ}(k)\right) \in \Upsilon$, the dead-beat control law (providing that $x(k+N)=x^{\circ}(k+N)$ )

$$
u(k)=K\left(x(k)-x^{\circ}(k)\right)+u^{\circ}(k)
$$

provides a feasible solution for $\left(x^{r}(k), \mathbf{u}^{r}(k)\right)=\left(x^{\circ}(k), \mathbf{u}^{\circ}(k)\right)$, resulting in an admissible predicted trajectory. Notice that the deadbeat control law exists since $N \geq n_{c}$ and the system is controllable as stated in Assumption 1.

Therefore, taking into account the optimality of the solution, for all $x(k)$ such that $\left(x(k)-x^{\circ}(k)\right) \in \Upsilon$, there exist a constant $c_{w}>0$ such that

$$
\begin{aligned}
W\left(x(k)-x^{\circ}(k)\right) & \leq \sum_{i=0}^{N-1}\left\|x(i ; k)-x^{\circ}(i ; k)\right\|_{Q}^{2} \\
& +\left\|u(i ; k)-u^{\circ}(i ; k)\right\|_{R}^{2}
\end{aligned}
$$

which is less or equal to

$$
\sum_{i=0}^{N-1} c_{w}\left\|\left(x(i ; k)-x^{\circ}(i ; k), u(i ; k)-u^{\circ}(i ; k)\right)\right\|^{2}
$$

Then taking into account the linearity of the system controlled with the dead-beat control law, there exists a constant $w_{c}>0$ such that

$$
\sum_{i=0}^{N-1}\left\|\left(x(i ; k)-x^{\circ}(i ; k), u(i ; k)-u^{\circ}(i ; k)\right)\right\|^{2} \leq w_{c}\left\|x(k)-x^{\circ}(k)\right\|^{2}
$$

Then we have that

$$
W\left(x(k)-x^{\circ}(k)\right) \leq c_{w} w_{c}\left\|x(k)-x^{\circ}(k)\right\|^{2}
$$

for all $\left(x(k)-x^{\circ}(k)\right) \in \Upsilon$.

Condition (iii): From standard arguments [1] and periodicity of $\mathbf{r}, \mathbf{y}^{r *}(k), \mathbf{u}^{r *}(k)$, the following inequalities follow:

$$
\begin{aligned}
& V_{N}^{*}(x(k), \mathbf{r}(k))-V_{N}^{*}(x(k-1), \mathbf{r}(k-1)) \\
\leq \quad & V_{N}\left(x(k), \mathbf{r}(k) ; x^{r s}(k), \mathbf{u}^{r s}(k), \mathbf{u}_{N}^{s}(k)\right) \\
& -V_{N}^{*}(x(k-1), \mathbf{r}(k-1)) \\
\leq \quad & -\left\|x^{*}(0 ; k-1)-x^{r *}(k-1)\right\|_{Q}^{2} \\
& -\left\|u_{N}^{*}(0 ; k-1)-u^{r *}(0 ; k-1)\right\|_{R}^{2} \\
& +V_{p}\left(\mathbf{r}(k) ; x^{r s}(k), \mathbf{u}^{r s}(k)\right) \\
& -V_{p}\left(\mathbf{r}(k-1) ; x^{r *}(k-1), \mathbf{u}^{r *}(k-1)\right) \\
\leq \quad & -\left\|x^{*}(0 ; k-1)-x^{r *}(k-1)\right\|_{Q}^{2} \\
& -\left\|u_{N}^{*}(0 ; k-1)-u^{r *}(0 ; k-1)\right\|_{R}^{2}
\end{aligned}
$$

From lemma 1 we have that there exist $x^{r}(k), \mathbf{u}^{r}(k), \mathbf{u}_{N}(k)$ such that

$$
V_{N}\left(x(k), \mathbf{r}(k) ; x^{r}(k), \mathbf{u}^{r}(k), \mathbf{u}_{N}(k)\right)-V_{N}^{*}(x(k-1), \mathbf{r}(k-1))
$$


is lees or equal to $-\gamma\left\|x(k-1)-x^{\circ}(k-1)\right\|^{2}$ for all $x(k-1) \in \mathcal{X}_{N}$ and some $\gamma>0$.

Lemma 1: If Problem $P_{N}(x(k-1) ; \mathbf{r}(k-1))$ is feasible, then there exists a positive constant $\gamma>0$ such that

$V_{N}^{*}(x(k), \mathbf{r}(k))-V_{N}^{*}(x(k-1) ; \mathbf{r}(k-1)) \leq-\gamma\left\|x(k-1)-\mathbf{x}^{\circ}(k-1)\right\|^{2}$

for all $x(k-1) \in \mathcal{X}_{N}$.

Proof: Consider that for $x(k)$ and the shifted reference $\left(x^{r s}(k), \mathbf{u}^{r s}(k)\right)$ the sequences introduced in Lemma 2 are defined. By feasibility we have that

$$
\left(x^{r s}(i ; k), u^{r s}(i ; k)\right) \in \mathcal{Z}^{c}
$$

and then there exists an $\epsilon>0$ such that if $\left\|x(k)-x^{r s}(k)\right\| \leq \epsilon$ then $\left(\mathbf{u}_{N}^{a}(k), x^{r s}(k), \mathbf{u}^{r s}(k)\right)$ is a feasible solution of $P_{N}(x(k), \mathbf{r}(k))$.

Take the constant $\Gamma_{k}=\left\|x^{r *}(k-1)-x^{\circ}(k-1)\right\|^{2}$ and let $\beta_{k} \in$ $(0,1)$ be a positive constant satisfying the conditions in Lemma 2 . Take a $\beta \in\left(\beta_{k}, 1\right)$ such that $(1-\beta)\left\|x^{r s}(k)-x^{\circ}(k)\right\| \leq \epsilon$ and define $\xi_{k}=(1-\beta)\left\|x^{r s}(k)-x^{\circ}(k)\right\|$. Then the following two cases are studied:

Case 1: $\left\|x(k)-x^{r s}(k)\right\| \geq \xi_{k}$.

By the definition of $x(k)$ and since $x^{r s}(k)=x^{r *}(1 ; k-1)$ we obtain

$$
\begin{aligned}
\xi_{k} \leq & \left\|x(k)-x^{r *}(1 ; k-1)\right\| \\
= & \| A\left(x(k-1)-x^{r *}(k-1)\right) \\
& +B\left(u^{*}(0 ; k-1)-u^{r *}(0 ; k-1)\right) \| \\
\leq & \rho\left\|\left(x(k-1)-x^{r *}(k-1)\right)\right\| \\
& +\rho\left\|\left(u^{*}(0 ; k-1)-u^{r *}(0 ; k-1)\right)\right\|
\end{aligned}
$$

where $\rho=\max \{\|A\|,\|B\|, 1\}$.

Consider the case that $\left\|\left(u^{*}(0 ; k-1)-u^{r *}(0 ; k-1)\right)\right\| \leq \frac{\xi}{2 \rho}$, then from the last inequality we have that

$$
\left\|\left(x(k-1)-x^{r *}(k-1)\right)\right\| \geq \frac{\xi}{2 \rho}
$$

and then

$$
\begin{aligned}
& \left\|\left(x(k-1)-x^{r *}(k-1)\right)\right\|_{Q}^{2} \\
& +\left\|\left(u^{*}(0 ; k-1)-u^{r *}(0 ; k-1)\right)\right\|_{R}^{2} \\
\geq & \lambda_{\min }(Q)\left\|x(k-1)-x^{r *}(k-1)\right\|^{2} \geq \frac{\lambda_{\min }(Q) \xi^{2}}{4 \rho^{2}}
\end{aligned}
$$

On the other hand, if we consider the case that

$$
\left\|\left(u^{*}(0 ; k-1)-u^{r *}(0 ; k-1)\right)\right\| \geq \frac{\xi}{2 \rho}
$$

then

$$
\begin{aligned}
& \left\|\left(x(k-1)-x^{r *}(k-1)\right)\right\|_{Q}^{2} \\
& +\left\|\left(u^{*}(0 ; k-1)-u^{r *}(0 ; k-1)\right)\right\|_{R}^{2} \\
\geq & \left\|\left(u^{*}(0 ; k-1)-u^{r *}(0 ; k-1)\right)\right\|_{R}^{2} \\
\geq & \lambda_{\min }(R)\left\|\left(u^{*}(0 ; k-1)-u^{r *}(0 ; k-1)\right)\right\|^{2} \geq \frac{\lambda_{\min }(R) \xi^{2}}{4 \rho^{2}}
\end{aligned}
$$

Let $\bar{\xi}:=\max \left\{\frac{\lambda_{\min }(Q) \xi^{2}}{4 \rho^{2}}, \frac{\lambda_{\min }(R) \xi^{2}}{4 \rho^{2}}\right\}$ and choose

$$
\gamma=\frac{\bar{\xi}}{\max \left\{\left\|x-x^{\circ}\right\|^{2} \mid x \in \mathcal{X}_{N},\left(x^{\circ}, u\right) \in \mathcal{Z}^{c}\right\}}
$$

Notice that constant $\gamma$ is positive and bounded since set $\mathcal{Z}$ is assumed to be compact.
Then,

$$
\begin{aligned}
&\left\|\left(x(k-1)-x^{r *}(k-1)\right)\right\|_{Q}^{2} \\
&+\left\|\left(u^{*}(0 ; k-1)-u^{r *}(0 ; k-1)\right)\right\|_{R}^{2} \\
& \geq \quad \bar{\xi}=\gamma \max \left\{\left\|x-x^{\circ}\right\|^{2}, x \in \mathcal{X}_{N},\left(x^{\circ}, u\right) \in \mathcal{Z}^{c}\right\} \\
& \geq \quad \gamma\left\|\left(x(k-1)-x^{\circ}(k-1)\right)\right\|^{2}
\end{aligned}
$$

Case 2: $\left\|x(k)-x^{r s}(k)\right\| \leq \xi_{k}$.

Consider the sequences defined in lemma 2 at $x(k)$, for the feasible shifted reference trajectory $\left(x^{r s}(k), \mathbf{u}^{r s}(k)\right)$ and taking the optimal unconstrained control law gain as the stabilizing control law gain $K$.

Since $\left\|x(k)-x^{r s}(k)\right\| \leq \xi_{k} \leq \epsilon$ and since the solution $\left(\mathbf{u}_{N}^{a}(k), x^{r s}, \mathbf{u}^{r s}(k)\right)$ is a feasible solutions of $P_{N}(x(k), \mathbf{r}(k))$, the solution $\left(\hat{\mathbf{u}}_{N}^{a}(k), \hat{x}^{r}, \hat{\mathbf{u}}^{r}(k)\right)$ is also feasible by convexity.

For the given $\Gamma_{k}$ and $\beta$, since

$$
\left\|x(k)-x^{r s}(k)\right\| \leq \xi_{k}=(1-\beta)\left\|x^{r s}(k)-x^{\circ}(k)\right\|
$$

we derive from lemma 2 that

$\hat{V}_{N}(x(k), \mathbf{r}(k)) \leq V_{N}(x(k), \mathbf{r}(k))-(1-\beta)^{2}\left\|x^{r *}(k-1)-x^{\circ}(k-1)\right\|^{2}$

Since $K$ is the optimal unconstrained control law, we have that

$$
V_{N}(x(k), \mathbf{r}(k)) \leq V_{N}\left(x(k), \mathbf{r}(k) ; x^{r s}(k), \mathbf{u}^{r s}(k), \mathbf{u}_{N}^{s}(k)\right)
$$

and then we have that

$$
\begin{aligned}
& \hat{V}_{N}(x(k), \mathbf{r}(k))-V_{N}^{*}(x(k-1), \mathbf{r}(k-1)) \\
\leq & V_{N}(x(k), \mathbf{r}(k))-(1-\beta)^{2} \| x^{r *}(k-1) \\
& -x^{\circ}(k-1) \|^{2}-V_{N}^{*}(x(k-1), \mathbf{r}(k-1)) \\
\leq & V_{N}\left(x(k), \mathbf{r}(k) ; x^{r s}(k), \mathbf{u}^{r s}(k), \mathbf{u}_{N}^{s}(k)\right) \\
& -V_{N}^{*}(x(k-1), \mathbf{r}(k-1)) \\
& -(1-\beta)^{2}\left\|x^{r *}(k-1)-x^{\circ}(k-1)\right\|^{2} \\
\leq \quad & -\left\|\left(x(k-1)-x^{r *}(k-1)\right)\right\|_{Q}^{2} \\
& -\left\|\left(u^{*}(0 ; k-1)-u^{r *}(0 ; k-1)\right)\right\|_{R}^{2} \\
& -(1-\beta)^{2}\left\|x^{r *}(k-1)-x^{\circ}(k-1)\right\|^{2} \\
\leq \quad & -\lambda_{\min }(Q)\left\|\left(x(k-1)-x^{r *}(k-1)\right)\right\|^{2} \\
& -(1-\beta)^{2} \| x^{r *}(k-1) \\
& -x^{\circ}(k-1) \|^{2} \\
\leq & -\gamma\left\|\left(x(k-1)-\mathbf{x}^{\circ}(k-1)\right)\right\|^{2}
\end{aligned}
$$

with $\gamma=\frac{1}{2} \min \left\{\lambda_{\min }(Q),(1-\beta)^{2}\right\}$.

Lemma 2: Let $x(k)$ be a given state and let $\left(x^{r}(k), \mathbf{u}^{r}(k)\right)$ be such that the associated trajectory is admissible. Let $\left(\mathbf{x}_{N}^{a}(k), \mathbf{u}_{N}^{a}(k)\right)$ be a sequence of states and control inputs derived from the control law $\kappa^{a}\left(x(i), x^{r}(k), \mathbf{u}^{r}(k)\right)=K\left(x(i)-x^{r}(i ; k)\right)+u^{r}(i ; k)$ such that $x^{a}(N ; k)=x^{r}(N ; k)$. Let $V_{N}(x(k), \mathbf{r}(k))$ be the cost associated to this solution, that is

$$
V_{N}(x(k), \mathbf{r}(k))=V_{N}\left(x(k), \mathbf{r}(k) ; \mathbf{u}_{N}^{a}(k), x^{r}(k), \mathbf{u}^{r}(k)\right)
$$

Let $\left(\hat{x}^{r}(k), \hat{\mathbf{u}}^{r}(k)\right)$ be defined as

$$
\left(\hat{x}^{r}(k), \hat{\mathbf{u}}^{r}(k)\right)=\beta\left(x^{r}(k), \mathbf{u}^{r}(k)\right)+(1-\beta)\left(x^{\circ}(k), \mathbf{u}^{\circ}(k)\right)
$$

and let $\hat{\mathbf{u}}_{N}^{a}(k)$ be a sequence of control inputs derived from the deadbeat control law $\kappa^{a}\left(x(i), \hat{x}^{r}(k), \hat{\mathbf{u}}^{r}(k)\right)=K\left(x(i)-\hat{x}^{r}(i ; k)\right)+$ $\hat{u}^{r}(i ; k)$. Let $\hat{V}_{N}(x(k), \mathbf{r}(k))$ be the cost associated to this solution, that is

$$
\hat{V}_{N}(x(k), \mathbf{r}(k))=V_{N}\left(x(k), \mathbf{r}(k) ; \hat{\mathbf{u}}_{N}^{a}(k), \hat{x}^{r}(k), \hat{\mathbf{u}}^{r}(k)\right)
$$


Then, for any positive constant $\Gamma>0$, there exists a constant $\beta \in(0,1)$ such that

$$
\left\|x(k)-x^{r}(k)\right\| \leq(1-\beta)\left\|x^{r}(k)-x^{\circ}(k)\right\|
$$

implies that $\hat{V}_{N}(x(k), \mathbf{r}(k))-V_{N}(x(k), \mathbf{r}(k)) \leq-(1-\beta)^{2} \Gamma$.

Proof: We denote $A_{c l}=A+B K$. From the definition of the dead-beat control law we obtain

$$
\begin{aligned}
& u^{a}(i ; k)=K A_{c l}^{i}\left(x(k)-x^{r}(k)\right)+\mathbf{u}^{r}(i ; k) \\
& x^{a}(i ; k)=A_{c l}^{i}\left(x(k)-x^{r}(k)\right)+x^{r}(i ; k)
\end{aligned}
$$

and similarly for $\hat{\mathbf{u}}_{N}^{a}(k) \hat{\mathbf{x}}_{N}^{a}(k)$. Taking into account (5), we can show that

$$
\begin{aligned}
& \sum_{i=0}^{N-1}\left\|\hat{x}^{a}(i ; k)-\hat{x}^{r}(i ; k)\right\|_{Q}^{2}+\left\|\hat{u}^{a}(i ; k)-\hat{u}^{r}(i ; k)\right\|_{R}^{2} \\
& -\sum_{i=0}^{N-1}\left(\left\|x^{a}(i ; k)-x^{r}(i ; k)\right\|_{Q}^{2}-\left\|u^{a}(i ; k)-u^{r}(i ; k)\right\|_{R}^{2}\right) \\
= & \left\|x(k)-\hat{x}^{r}(k)\right\|_{H}^{2}-\left\|x(k)-x^{r}(k)\right\|_{H}^{2} \\
= & \left\|x(k)-\mathrm{x}^{r}(k)+(1-\beta)\left(x^{r}(k)-x^{\circ}(k)\right)\right\|_{H}^{2} \\
& -\left\|x(k)-x^{r}(k)\right\|_{H}^{2} \\
= & (1-\beta)^{2}\left\|x^{r}(k)-\mathbf{x}^{\circ}(k)\right\|_{H}^{2} \\
& +2(1-\beta)(x(k) \\
& \left.\left.-x^{r}(k)\right)^{T} H\left(x^{r}(k)-x^{\circ}(k)\right)\right) \\
\leq \quad & (1-\beta)^{2}\left\|x^{r}(k)-x^{\circ}(k)\right\|_{H}^{2} \\
& +2(1-\beta)\|H\|\left\|x(k)-x^{r}(k)\right\|\left\|x^{r}(k)-x^{\circ}(k)\right\| \\
\leq \quad & (1-\beta)^{2} \lambda_{H}\left\|x^{r}(k)-x^{\circ}(k)\right\|^{2} \\
& +2(1-\beta) \lambda_{H}\left\|x(k)-x^{r}(k)\right\|\left\|x^{r}(k)-x^{\circ}(k)\right\| \\
\leq \quad & (1-\beta)^{2} \lambda_{H}\left\|x^{r}(k)-x^{\circ}(k)\right\|^{2} \\
& +2(1-\beta)^{2} \lambda_{H}\left\|x^{r}(k)-x^{\circ}(k)\right\|^{2} \\
= & 3(1-\beta)^{2} \lambda_{H}\left\|x^{r}(k)-x^{\circ}(k)\right\|^{2}
\end{aligned}
$$

where $H$ is defined as

$$
H=\sum_{i=0}^{N-1} A_{c l}^{i^{T}}\left(Q+K^{T} R K\right) A_{c l}^{i}
$$

which is positive definite. The constant $\lambda_{H}=\lambda_{\max }(H)$. Convexity of $V_{p}(\cdot)$ provides that

$$
V_{p}\left(\mathbf{r}(k), \hat{x}^{r}(k), \hat{\mathbf{u}}^{r}(k)\right) \leq \beta V_{p}\left(\mathbf{r}(k), x^{r}(k), \mathbf{u}^{r}(k)\right)+(1-\beta) V_{p}^{\circ}
$$

Using these results, it can then be seen that for any $\Gamma>0$

$$
\begin{aligned}
& \hat{V}_{N}(x(k), \mathbf{r}(k))-V_{N}(x(k), \mathbf{r}(k))+(1-\beta)^{2} \Gamma \\
\leq \quad & (1-\beta)^{2} \Gamma+3(1-\beta)^{2} \lambda_{H}\left\|x^{r}(k)-x^{\circ}(k)\right\|^{2} \\
& -(1-\beta)\left(V_{p}\left(\mathbf{r}(k), x^{r}(k), \mathbf{u}^{r}(k)\right)-V_{p}^{\circ}\right)
\end{aligned}
$$

Since $V_{p}\left(\mathbf{r}(k) ; x^{r}(k), \mathbf{u}^{r}(k)\right)>V_{p}^{\circ}$ by optimality of the optimal reachable reference, for any $\Gamma>0$ there exists a $\beta \in(0,1)$, such that

$$
\hat{V}_{N}(x(k), \mathbf{r}(k))-V_{N}(x(k), \mathbf{r}(k))+(1-\beta)^{2} \Gamma \leq 0
$$

\section{EXAMPLE}

In this section we apply the proposed controller to a linear approximation of a ball and plate system. The system consists of a plate pivoted at its center such that the slope of the plate can be manipulated in two perpendicular directions. A servo system consisting of motors

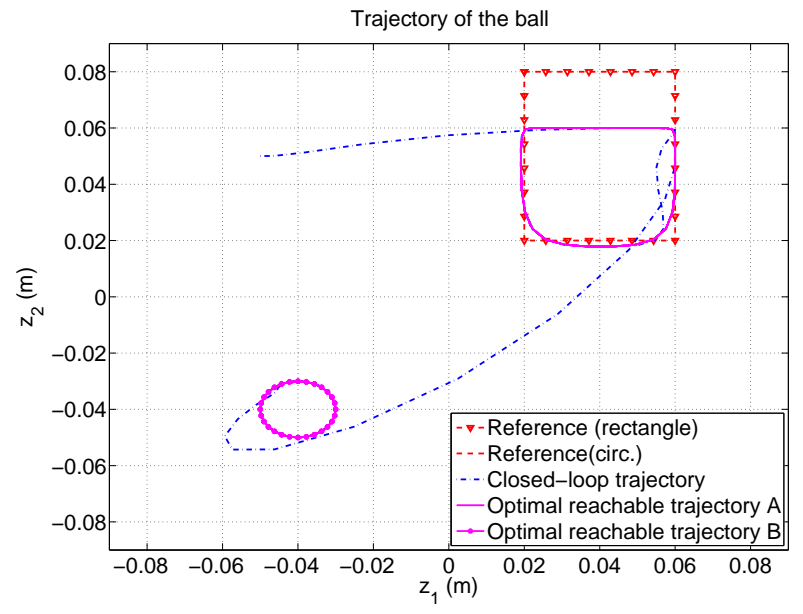

Fig. 1. Trajectories of $z_{1}, z_{2}$ for the closed loop system (dash-dotted blue), the trajectory planner (solid magenta) and the target reference (dashed red) (scenario 2).

is used for tilting the plate and control the two angles of rotation $\theta_{1}, \theta_{2}$. An appropriate sensor for measurement of the ball position $z_{1}, z_{2}$ is assumed to be available, for example an intelligent vision system. The basic control task is to control the position of a ball freely rolling on a plate. This system is a dynamic system with two inputs and two outputs.

To carry out the simulations a discrete time linear system is obtained taking as equilibrium point the origin for all the states and inputs and a sampling time $T m=0.05$ seconds, see details in [14],[15]. This system satisfies Assumption (1). The inputs of the ball and plate system are the accelerations applied in each rotation axis and they are denoted as $\mathcal{U}=\left[u_{1}, u_{2}\right]^{t}=\left[\ddot{\theta}_{1}, \ddot{\theta}_{2}\right]^{t}$. The state $x \in R^{8}$ is defined as follows

$$
x^{T}=\left[z_{1}, \dot{z}_{1}, \theta_{1}, \dot{\theta}_{1}, z_{2}, \dot{z}_{2}, \theta_{2}, \dot{\theta}_{2}\right]^{T}
$$

The system must satisfy $\left|z_{i}\right| \leq 6 \mathrm{~cm},\left|\theta_{i}\right| \leq \frac{\pi}{2} \mathrm{rad}$ and $\left|\ddot{\theta}_{i}\right| \leq$ $110 \mathrm{rad} / \mathrm{s}^{2}$

In the simulation a short prediction horizon $N=5$ is chosen to demonstrate that the proposed controller has a large domain of attraction that has a low dependence on the prediction horizon $N$. The number of decision variables is $n_{u} \cdot(N+T)+n_{x}=74$. In addition, in order to prove that recursive feasibility is not lost even in the presence of a sudden change in the target reference, the reference switches between two geometric figures. First the ball must draw a rectangle of size $6 \times 4 \mathrm{~cm}$ that is centered in $(4,5) \mathrm{cm}$ with a speed of $11.43 \frac{\mathrm{cm}}{\mathrm{s}}$. At time 2.8 seconds the reference changes in order to draw a circumference with center $(-4,-4) \mathrm{cm}$ and a radius of $1 \mathrm{~cm}$. The target speed of the second trajectory is $2.3 \frac{\mathrm{cm}}{\mathrm{s}}$. The period length of both references is the same, that is $T=28$. The initial state of this scenario is the ball in equilibrium at $\left(z_{1}, z_{2}\right)=(-5,5) \mathrm{cm}$.

The simulation shows that when the reference changes suddenly, the trajectory of the ball converges to the new trajectory of the planner satisfying the constraints and without losing feasibility even when the prediction horizon is much lower than the period length. Figure 1 shows the trajectories of $z_{1}, z_{2}$ for the closed loop system (dash-dotted blue), the trajectory planner (solid magenta) and the target reference (dashed red) in the $z_{1}, z_{2}$ plane. Figures 2,3 show the trajectory of the ball on each axis. In these figures, it can be seen that the trajectory of the closed-loop system converges to the optimal reachable reference trajectories with zero error, first to the trajectory planner of the rectangle, and then to the trajectory planner 


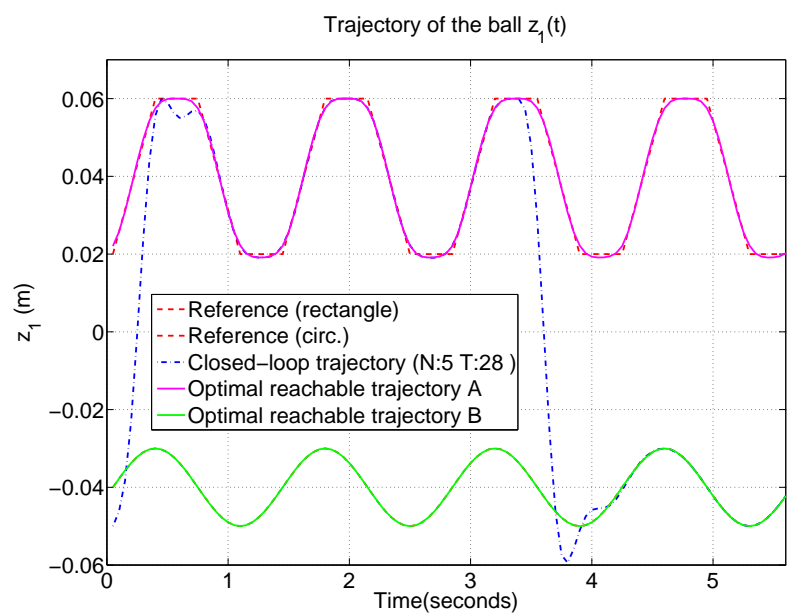

Fig. 2. Trajectories of $z_{1}$ for the closed loop system (dash-dotted blue), the trajectory planner (solid magenta) and the target reference (dashed red) (scenario 2).

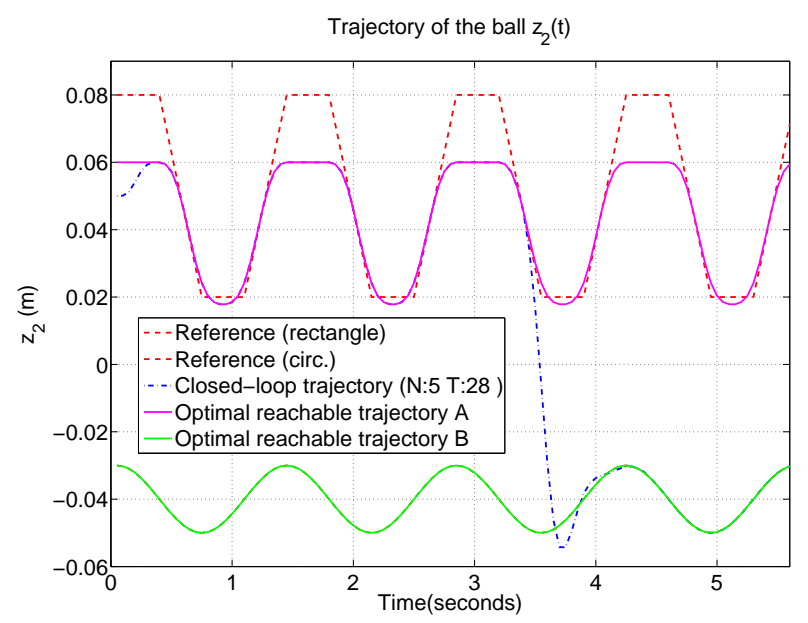

Fig. 3. Trajectories of $z_{2}$ for the closed loop system (dash-dotted blue), the trajectory planner (solid magenta) and the target reference (dashed red) (scenario 2).

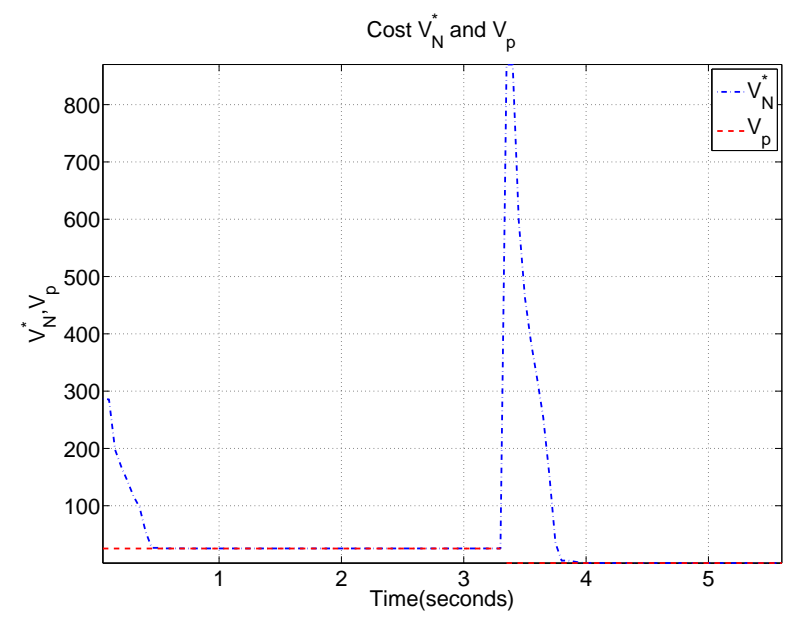

Fig. 4. Trajectories of the optimal cost $V_{N}^{*}$ (dashed blue) and trajectory planner cost $V_{p}^{o}$ (dashed red) (scenario 1). of the circle. All these figures show a sudden change in when the reference switches from the rectangle to the circle. It can be seen that there exists a deviation between the trajectory of the planner and the target reference for the rectangle, but that the error is zero for the circle reference, which is reachable. For this reason the optimal cost of the optimization problem (2) is non-zero for the rectangle and zero for the circle. Figure 4 shows that the cost of the proposed controller converges to the cost of the trajectory planners in a nonincreasing manner, demonstrating that the difference between both values increases suddenly when the reference changes, but that then it converges again to the new optimal trajectory planner cost. It is important to remark, that when the target reference changes, all the state variables are far away from the optimal reachable reference, and that they take more than 5 time steps to reach it, however, the MPC maintains feasibility as proved in Theorem 2 .

\section{REFERENCES}

[1] J. B. Rawlings and D. Q. Mayne, Model Predictive Control: Theory and Design, 1st ed. Nob-Hill Publishing, 2009.

[2] E. F. Camacho and C. Bordons, Model Predictive Control, 1st ed. Springer-Verlag, 1999.

[3] J. Lee, S. Natarajan, and K. Lee, "A model-based predictive control approach to repetitive continuous processes with periodic operations," Journal of Process Control, vol. 11, pp. 195-207, 2001.

[4] B. Kern, C. Bhm, R. Findeisen, and F. Allgwer, "Receding horizon control for linear periodic time-varying systems subject to input constraints," in Nonlinear Model Predictive Control, ser. Lecture Notes in Control and Information Sciences, L. Magni, D. Raimondo, and F. Allgwer, Eds. Springer Berlin Heidelberg, 2009, vol. 384, pp. 109117.

[5] R. Gondhalekar, F. Oldewurtel, and C. N. Jones, "Least-restrictive robust periodic model predictive control applied to room temperature regulation," Automatica, vol. 49, pp. 2760-2766, 2013.

[6] R. Huang, E. Harinath, and L. T. Biegler, "Lyapunov stability of economically oriented NMPC for cyclic processes," Journal of Process Control, vol. 21, pp. 501-509, 2011.

[7] L. Magni, G. De Nicolao, and R. Scattolini, "Output feedback and tracking of nonlinear systems with model predictive control." Automatica, vol. 37, pp. 1601-1607, 2001.

[8] U. Mäder and M. Morari, "Offset-Free Reference Tracking with Model Predictive Control," Automatica, vol. 46, no. 9, pp. 1469-1476, 2010.

[9] H. Ito, C. Nakazawa, T. Matsui, K. Matsumoto, and H. Nishida, "Model predictive control for a periodic reference signal," in In Proc. SICE Annual Conference 2007, 2007, pp. 2575-2577.

[10] D. Limon and T. Alamo, "Tracking model predictive control," in Encyclopedia of Systems and Control, J. Baillieul and T. Samad, Eds. Springer, 2014.

[11] A. Ferramosca, D. Limon, I. Alvarado, T. Alamo, and E. F. Camacho, "MPC for tracking with optimal closed-loop performance," Automatica, vol. 45, pp. 1975-1978, 2009.

[12] D. Limon, I. Alvarado, T. Alamo, and E. F. Camacho, "MPC for tracking of piece-wise constant references for constrained linear systems," Automatica, vol. 44, pp. 2382-2387, 2008.

[13] C. V. Rao and J. B. Rawlings, "Steady states and constraints in model predictive control," AIChE Journal, vol. 45, pp. 1266-1278, 1999.

[14] M. A. Moreno-Armendáriz, C. A. Pérez-Olvera, F. O. Rodríguez, and E. Rubio, "Indirect hierarchical FCMAC control for the ball and plate system," Neurocomputing, vol. 73, pp. 2454-2463, 2010.

[15] Y. Wang, M. Sun, Z. Wang, Z. Liu, and Z. Chen, "A novel disturbanceobserver based friction compensation scheme for ball and plate system," ISA Transactions, vol. 53, pp. 671-678, 2014. 